Souza, Rayse Kiane de; Souza, Márcio Vieira de; "ANÁLISE DE USABILIDADE EM MOOCS (MASSIVE OPEN ONLINE COURSES): UMA ABORDAGEM QUALITATIVA", p. 139-152 . In: Souza, Márcio Vieira de; Giglio, Kamil. Mídias Digitais, Redes Sociais e Educação em Rede: Experiências na Pesquisa e Extensão Universitária. São Paulo: Blucher, 2015. ISBN: 978-85-8039-128-2, DOI 10.5151/9788580391282-12

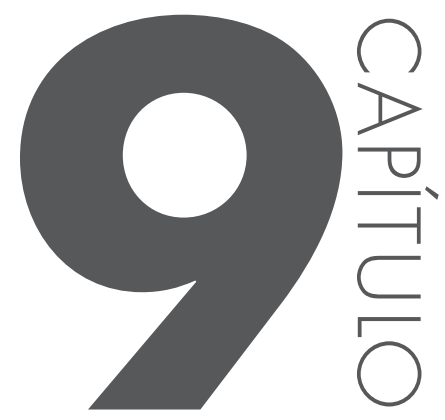

\title{
ANÁLISE DE USABILIDADE EM MOOCS (MASSIVE OPEN ONLINE COURSES): UMA ABORDAGEM QUALITATIVA7
}

Rayse Kiane de SOUZA; Márcio Vieira de SOUZA

\section{INTRODUC̣ÃO}

No desenvolvimento de softwares a busca da qualidade envolve diversos aspectos na produção, desde funcionalidades, confiabilidade e segurança de uso, até as chamadas qualidades extras ou implícitas como flexibilidade, adaptabilidade e facilidade de entendimento. Dentro desse conjunto de critérios está a interface que é responsável pela interação do usuário com o computador a qual deve primar pela qualidade da comunicação entre ambos.

No contexto de AVA e MOOCs, temos um universo inteiro de tipos de usuários, dos mais avançados, que já nasceram na era da internet, até

${ }^{7}$ Esse texto tem como base o TCC de Rayse Kiane de Souza defendido em 2014 no curso de Sistemas de Informação da UFSC, sob orientação do prof. Dr. Márcio Vieira de Souza. 
os mais novos ingressantes deste mundo com poucas habilidades. E é pensando em todos os tipos de usuário que estas plataformas abrangem, ou almejam abranger, que a usabilidade se torna imprescindível. Uma interface mal planejada, que não seja amigável e eficiente, pode ser responsável pela desistência de estudantes em ambientes de aprendizagem massivos, um dos maiores problemas envolvendo esse tipo de plataforma.

Para Nielsen (1993), usabilidade de sistemas está totalmente ligada a estes cinco fatores: instintividade, eficiência, memorização, erros e satisfação. E são estes fatores que este trabalho, através de uma análise de uso, pretende qualificar estes cinco MOOCs: Coursera, edX, MiríadaX, Udacity e Veduca.

Como explicado anteriormente, diferente dos AVA tradicionais, que servem para dar apoio às disciplinas presenciais ou fazerem cursos fechados totalmente virtualizados, os MOOCs (Massive Open Online Courses) aparecem com o princípio de massificar estes cursos via rede, sendo, portanto, abertos a todos os usuários que se propuserem a realizar o curso. Este conceito de “aberto", segundo Mattar (2013b) já é bastante problemático, pois em muitos casos uma taxa é cobrada caso o estudante deseje receber um certificado de participação. Por outro lado, o conhecimento ainda permanece aberto, mesmo que, conforme o autor alerta exista a tendência de que algumas MOOCs passem a serem pagos em um futuro não muito distante.

Segundo Mattar (2013b), muitas dessas plataformas são baseadas no conceito de anonimato dos movimentos Open Course Ware. Sendo assim, as interações entre estudantes e equipe pedagógica são bastante enfraquecidas. Outra característica diz respeito a duração dos cursos, que em geral são de grande duração, tornando-se um fator desmotivador aos participantes. Esse aspecto pode ser percebido em um dos cursos oferecidos pela Universidade de Stanford, onde dos 160 mil inscritos apenas $15 \%$ concluíram o curso em sua totalidade (SIEMENS, 2013).

Contando com a interação dos usuários, as cMOOCs, que segundo McAuley (2010) são construídos pelo envolvimento ativo dos estudantes, que auto organizam sua participação em função de seus objetivos de aprendizagem, conhecimento prévios e interesses comuns. Por este motivo, possuem pouca estrutura, comparados a outros cursos online, redefinindo assim a própria noção de curso e relação entre educando e educando e educador. De acordo com Mattar (2013b) este é o motivo pela qual as MOOCs possibilitam aprendizagem social e informal interativa com baixo custo e oferecida em larga escala. 


\section{USABILIDADE}

Segundo o renomado especialista em usabilidade Jakob Nielsen, usabilidade foi definida como "A usabilidade é um atributo de qualidade que avalia quão fáceis interfaces de usuário são para usar". Nielsen usa o termo usabilidade para se denotar as considerações que podem ser abordadas pelos métodos propostos por ele que são usados durante o processo de design para melhorar a facilidade de uso.

A usabilidade tem como objetivo elaborar interfaces capazes de permitir uma interação fácil, agradável, com eficácia e eficiência. Ela deve capacitar a criação de interfaces transparentes de maneira a não dificultar o processo, permitindo ao usuário pleno controle do ambiente sem se tornar um obstáculo durante a interação.

De acordo com Nielsen, usabilidade é tradicionalmente associada a estes cinco atributos:

INTUITIVIDADE: O sistema deve ser fácil de usar, de modo que um novo usuário consiga ter uma produtividade satisfatória.

EFICIÊNCIA: O sistema deve ser eficiente para utilizar, de modo que uma vez que o utilizador tenha aprendido o sistema, um alto nível de produtividade é possível.

MEMORIZAÇÃo: As telas do sistema devem apresentar facilidade de memorização permitindo que usuários ocasionais consigam utilizá-lo mesmo depois de um longo intervalo de tempo.

ERROS: O sistema deve ter uma baixa taxa de erro, caso o ocorra algum deve fornecer uma fácil recuperação a partir deles. Além disso, erros catastróficos não devem ocorrer.

SATISFAÇÃO: O sistema deve satisfazer o usuário, sejam eles iniciantes ou avançados, permitindo uma interação agradável.

\section{AVALIAC̣ÃO DE USABILIDADE}

Avaliações de usabilidade possibilitam detectar e corrigir problemas de usabilidade específicos, a fim de melhorar a interação dos usuários. Diretrizes de usabilidade têm tipicamente milhares de regras a seguir e, portanto, são vistas como intimidantes pelos desenvolvedores.

Segundo Dias (2007), existem três grupos de métodos para avaliação de usabilidade: 
I. MÉTODOS DE INSPEÇÃo: Os métodos de inspeção podem ser divididos em Métodos Analíticos ou Prognósticos e são aplicados apenas por especialistas. Geralmente os avaliadores que adotam esses métodos são especialistas em usabilidade ou projetistas que se baseiam em regras e recomendações, princípios e/ou conceitos pré-estabelecidos para verificar problemas de usabilidade. Os principais métodos de inspeção são: Inspeção de Usabilidade Formal, Inspeção ou Percurso Pluralístico, Inspeção de Componentes, Inspeção de Consciência, Inspeção ou Percurso Cognitivo, Inspeção Baseada em Padrões, Inspeção Baseada em Guias de Recomendações e Guias de Estilos e Avaliação Heurística.

2. MÉTODOS DE TESTE COM O USUÁRIO: O usuário do sistema participa ativamente na avaliação. Os principais métodos de testes com os usuários são: Entrevistas e Questionários, Grupo Focal, Questionários Específicos para Medir a Satisfação dos Usuários, Técnicas Empíricas de Usabilidade, Verbalização ou Protocolo Verbal, Co-descoberta e Método de Medida de Desempenho.

3. MÉTOdos baseados EM modelos: Têm como objetivo prever a usabilidade de um sistema a partir de modelos ou representações de sua interface e/ou de seus usuários. Esses métodos pretendem representar como os usuários interagem com um sistema.

A avaliação heurística, método de inspeção que foi aplicado neste trabalho, é utilizada por arquitetos de informação e designers de interação para realizar testes de usabilidade em interfaces de modo rápido, barato e fácil, assim sendo um método simples que pode ser aplicado por qualquer equipe de desenvolvimento, ou mesmo podendo ser realizado posteriormente a sua aplicação para testar se o ambiente se adequa as exigências dos usuários.

Para simplificar o uso dos princípios de usabilidade durante o design de um sistema, Nielsen propôs um conjunto de 10 heurísticas gerais de usabilidade que são amplamente utilizados neste tipo de avaliação. Para Nielsen, "o objetivo da avaliação heurística é encontrar os problemas de utilização na concepção de modo que eles podem ser atendidos como parte de um processo iterativo de design”.

Heurísticas são regras gerais que descrevem uma propriedade comum, neste caso derivadas de conhecimento de aspectos psicológicos, computacionais, e sociológicos dos domínios do problema. São independentes de uma tecnologia específica e são resultantes de uma base de problemas comuns entre muitos sistemas. As 10 Heurísticas de Usabilidade, por Jakob Nielsen são:

I. VISIBILIDADE DO STATUS DO SISTEMA: O sistema deve sempre manter os usuários informados sobre o que está acontecendo, através de feedback apropriado em tempo razoável. 
2. CORRESPONDÊNCIA ENTRE O SISTEMA E O MUNDO REAL: O sistema deve falar a linguagem dos usuários, com palavras, frases e conceitos familiares ao usuário, ao invés de termos orientados ao sistema. Deve seguir convenções do mundo real, tornando as informações que aparecem em uma ordem natural e lógica.

3. CONTROLE DO USUÁRIO E LIBERDADE: Usuários frequentemente escolhem algumas funções do sistema por engano e vão precisar sempre de uma "saída de emergência" claramente marcada para sair daquele estado indesejado sem ter que passar por um extenso "diálogo". Deve fornecer suporte para desfazer e refazer.

4. CONSISTÊNCIA E PADRÕES: Os usuários não precisam adivinhar que diferentes palavras, situações ou ações significam a mesma coisa. Siga os padrões da plataforma, padrões de interface, tanto em forma, cores e posições.

5. PREVENÇÃo DE ERROS: Ainda melhor do que boas mensagens de erro é um projeto cuidadoso que impede que em primeiro lugar esse erro possa ocorrer. Eliminar as condições passíveis de erros e verifica-las, apresentado aos usuários uma opção de confirmação antes de se comprometerem com uma determinada ação.

6. RECONHECIMENTO EM VEZ DE RECORDAÇÃo: Minimizar a carga de memória do usuário tornando objetos, ações e opções visíveis. O usuário não deve ter que se lembrar da informação de uma parte do diálogo para outra. Instruções de uso do sistema devem estar visíveis e serem facilmente recuperáveis quando necessário.

7. FLEXIBILIDADE E EFICIÊNCIA DE UTILIZAÇÃO: Aceleradores - invisíveis para o usuário novato -podem frequentemente acelerar a interação para o usuário experiente, o sistema pode atender a ambos os usuários inexperientes e experientes. Permitir aos usuários personalizar ações frequentes.

8. eSTÉTICA E DESIGN MINIMALISTA: Os diálogos não devem conter informações irrelevantes ou raramente necessárias. Cada unidade extra de informação em um diálogo compete com as unidades relevantes de informação e diminui sua visibilidade relativa.

9. AJUDE OS USUÁRIOS A RECONHECER, DIAGNOSTICAR E RESOLVER ERROS: Mensagens de erros devem ser expressas em linguagem clara (sem códigos), indicar com precisão o problema e construtivamente sugerir uma solução.

IO. AJUDA E DOCUMENTAÇÃO: Mesmo que seja melhor que um sistema possa ser usado sem documentação, pode ser necessário fornecer uma ajuda e documentação. Qualquer informação deve ser fácil de ser pesquisada com foco na atividade do usuário, deve fornecer uma lista de passos concretos a serem realizados e não ser muito grande. 
Utilizando-se da técnica de avaliação heurística é possível fazer uma classificação da severidade dos problemas encontrados, atribuindo-lhes uma nota. Estes erros são julgados com base em frequência, persistência e impacto na tarefa. São classificados da seguinte forma:

o. Viola uma heurística, mas parece não ser um problema de usabilidade.

I. Problema superficial (cosmético): pode ser facilmente superado pelos usuários ou acontece extremamente infrequentemente.

2. Problema menor de usabilidade: pode acontecer mais frequentemente ou ser mais difícil de superar.

3. Problema maior de usabilidade/ importante de corrigir: acontece frequentemente ou usuários não podem superar o problema.

4. Catástrofe de usabilidade/ imperativo de corrigir: seriamente impede o uso do sistema e não pode ser superado pelos usuários.

Para cada tarefa ou tela do sistema é necessário realizar uma inspeção passando por todas as heurísticas que se deseja avaliar. E os erros encontrados devem categorizados e proposta uma solução para eles.

\section{MÉTODO E ANÁLISE COMPARATIVA ENTRE PLATAFORMAS}

Os MOOCs por serem massivos, abrangem um número muito grande de usuários, entre eles usuários experientes e iniciantes. Por isso devem possuir características que acomodem ambos os tipos de usuários que o procuram. Mais que isso, devem ser atrativos e não intimidadores para os usuários que procuram este tipo de sistemas pela primeira vez, pois o MOOC tem o objetivo de disseminação de conhecimento, e procura atingir o maior número de pessoas possivel.

Com esta ideia foram avaliadas, de acordo com as heurísticas de Nielsen, as seguintes telas/tarefas de sistema:

- Tela inicial;

- Busca de curso;

- Cadastro de usuário;

- Cursar uma aula.

Este trabalho utilizou o método de avaliação heurística proposto por Nielsen (1993). Como proposto por Souza, R. (2014) cada tarefa foi avaliada de acordo com as dez heurísticas e foram levantados pontos positivos e negativos 
em cada uma delas, para isso utilizou como guia o checklist desenvolvido por Deniese Pierotti. O checklist contempla as 10 heurísticas de Nielsen e faz até 30 afirmações para cada uma, que devem ser respondidas com sim, não ou não se aplica. Por se tratar de um trabalho qualitativo um sistema de conceitos foi formulado para avaliar as plataformas.

Este método de conceitos foi inspirado no sistema SUS (System Usability Scale) de avaliação e na classificação dos erros. O SUS é essencialmente um método que trabalha com a avaliação do usuário final, este trabalho não realizou testes com usuários apenas se inspirou nos itens que o mesmo usa, como nível de facilidade de uso, nível de suporte, grau de aprendizado necessário para utilizar o sistema etc.

De acordo com número de erros, padronização, número de passos para concluir a tarefa, grau de documentação e ajuda e acessibilidade para qualquer nível de usuário os sistemas foram classificados como péssimo, ruim, bom ou ótimo.

Deste modo, todos os testes realizados foram executados com usuários do tipo estudante, disponível a todos. Este trabalho não contempla uma avaliação sobre os materiais e conteúdos disponibilizados pelos MOOCs, somente como os sistemas se apresentam e funcionam. Os sistemas que oferecem a opção de exibição em português, estes assim foram avaliados e a qualidade da tradução considerada, os demais foram avaliados no idioma nativo. Para cada tarefa escolhida uma tabela de heurísticas que foram violadas é apresentada, e para a tarefa de assistir uma aula, também, as principais características de cada um.

\section{TELA INICIAL}

A avaliação das telas iniciais foi realizada simulando um novo usuário, logo, o login de estudante não foi realizado.

Tabela 1 - Avaliação da Tela Inicial

\begin{tabular}{cccc}
\hline PLATAFORMAS & \multicolumn{2}{c}{ HEURÍSTICAS VIOLADAS } & CLASSIFICAÇÃO \\
& CONSISTÊNCIA E PADRÃO & ESTÉTICA E DESIGN MINIMALISTA & \\
\hline COURSERA & $X$ & & ÓTIMA \\
\hline EDX & $X$ & $X$ & BOA \\
\hline MIRÍADAX & $X$ & BOA \\
\hline UDACITY & & & ÓTIMA \\
\hline VEDUCA & $X$ & BOA \\
\hline
\end{tabular}

Fonte: Souza (2014) 
Os principais problemas encontrados na página inicial foram relativos a tradução das páginas, nas plataformas que disponibilizavam. No caso do edX, foi observado um layout de página que não favorecia a legibilidade das informações, por isso a quebra da heurística de design.

\section{CADASTRO DE NOVO USUÁRIO}

Três das plataformas deixaram a dever a respeito da ajuda ao usuário, não dando informações necessárias ou auxilio para preencher os formulários. As demais, apresentaram um padrão diferente de design na tela de cadastro, algo não recomendável, pois pode confundir usuários menos experientes.

\section{Tabela 2 - Avaliação Cadastro de Novo Usuário}

\begin{tabular}{|c|c|c|c|}
\hline \multirow[t]{2}{*}{ PLATAFORMAS } & \multicolumn{2}{|c|}{ HEURÍSTICAS VIOLADAS } & \multirow[t]{2}{*}{ CLASSIFICAC̣̃̃O } \\
\hline & CONSISTÊNCIA E PADRÃO & AJUDA E DOCUMENTAÇÃO & \\
\hline COURSERA & & & ÓTIMA \\
\hline EDX & & $x$ & $\mathrm{BOA}$ \\
\hline MIRÍADAX & $x$ & & $\mathrm{BOA}$ \\
\hline UDACITY & & $x$ & $\mathrm{BOA}$ \\
\hline VEDUCA & $x$ & $x$ & RUIM \\
\hline
\end{tabular}

Fonte: Souza (2014) 


\section{BUSCA DE CURSO}

Todas, com exceção do Veduca, apresentaram ótimos sistemas de busca. O MiríadaX obteve uma avaliação inferior pois seu sistema de busca não possuía busca por palavra-chave, somente por categorias. E o Veduca se mostrou o mais fraco nesta área, com layout diferente das demais telas, pouca ajuda ao usuário e um mecanismo de busca com poucos recursos.

Tabela 3 - Avaliação de Busca de Curso

\begin{tabular}{|c|c|c|c|c|}
\hline \multirow[t]{2}{*}{ PLATAFORMAS } & \multicolumn{3}{|c|}{ HEURISTIICAS VIOLADAS } & \multirow[t]{2}{*}{ CLASSIFICAC̣̃̃O } \\
\hline & $\begin{array}{l}\text { CONSISTÊNCIA } \\
\text { E PADRÃO }\end{array}$ & $\begin{array}{l}\text { AJUDA E } \\
\text { DOCUMENTAC̄̃̃ }\end{array}$ & $\begin{array}{l}\text { CONTROLE E } \\
\text { LIBERDADE }\end{array}$ & \\
\hline COURSERA & & & & ÓTIMA \\
\hline EDX & & & & о́тIMO \\
\hline MIRÍADAX & & & $x$ & BOA \\
\hline UDACITY & & & & о́тімо \\
\hline VEDUCA & $x$ & $x$ & $x$ & RUIM \\
\hline
\end{tabular}

Fonte: Souza (2014)

\section{AULA}

Para avaliar a aula, ou um módulo de um curso de cada sistema, foi escolhido previamente um curso que estivesse disponível durante o período de realização deste trabalho.

\section{Tabela 4 - Avaliação de Aula}

\begin{tabular}{|c|c|c|c|}
\hline \multirow[t]{2}{*}{ PLATAFORMAS } & \multicolumn{2}{|c|}{ HEURISTICAS VIOLADAS } & \multirow[t]{2}{*}{ CLASSIFICAC̣̃̃O } \\
\hline & VISIBILIDADE DO SISTEMA & AJUDA E DOCUMENTAÇÃO & \\
\hline COURSERA & & & ÓTIMA \\
\hline EDX & & & ÓtIMO \\
\hline MIRÍADAX & & $x$ & $\mathrm{BOA}$ \\
\hline UDACITY & & & ÓtIMO \\
\hline VEDUCA & $X$ & & $B O A$ \\
\hline
\end{tabular}

Fonte: Souza (2014) 
Os pouco problemas de usabilidade encontrados foram, primeiramente no MiríadaX, a falta de documentação e tutorias para cursar as aulas. E no Veduca algumas tarefas dos sistemas que demoram um tempo desnecessário para serem executadas, como por exemplo salvar uma nota, quebrando a heurística de visibilidade do sistema.

Foram levantadas as ferramentas que cada sistema oferece como suporte ao aprendizado dos estudantes, quais os seus diferenciais e avaliada a usabilidade para completar um modulo. Todos os cursos escolhidos são da modalidade gratuita e estavam disponíveis entre os meses de setembro e novembro de 2014.

\section{Tabela 5: Recursos Aulas}

\begin{tabular}{|c|c|c|c|c|c|}
\hline $\begin{array}{l}\text { PLATAFORMAS/ } \\
\text { RECURSOS }\end{array}$ & COURSERA & EDX & MIRÍADAX & UDACITY & VEDUCA \\
\hline VÍDEO AULA & $\operatorname{SIM}^{*}$ & $\operatorname{SIM}^{*}$ & SIM & SIM* $^{*}$ & SIM \\
\hline $\begin{array}{l}\text { LEGENDA/ } \\
\text { TRANSCRIÇÃO }\end{array}$ & $\operatorname{SIM}^{*}$ & $\operatorname{SIM}^{*}$ & SIM & $\operatorname{SIM}^{*}$ & SIM \\
\hline $\begin{array}{l}\text { TEXTOS/SLIDES } \\
\text { PARA DOWNLOAD }\end{array}$ & SIM & SIM & NÃO & SIM & NÃO \\
\hline EXERCíCIOS & SIM & SIM & SIM & SIM & NÃO \\
\hline FÓRUNS & SIM & SIM & SIM & SIM & SIM \\
\hline
\end{tabular}

Fonte: Souza (2014)

*Disponivel para download.

Deste modo, o intuito das cinco diferentes plataformas MOOC analisadas foi verificar a usabilidade das principais tarefas de cada uma e realizar um levantamento das principais características das mesmas.

Ao verificar as plataformas de acordo com as heurísticas de usabilidade foi possível analisar os problemas que usuários do sistema se deparam durante o uso das mesmas. Como estes ambientes tem um grande número de usuários, sendo estes com capacidades e conhecimentos diferentes, a ferramenta deve possuir um perfil simples e fácil utilização, para abranger o maior número de pessoas possível. Os problemas encontrados, design inconsistentes, erros de tradução, mecanismos de busca mal projetados, desmotivam o usuário a se cadastrar e a testar e incentivam a desistência dos que já são estudantes, um dos maiores problemas dos grandes MOOCs. Estes erros encontrados são de fácil solução, e se corrigidos poderiam melhor significativamente a experiência e a forma de adquirir o conhecimento transmitido pelo usuário. 
Os MOOCs apresentados são casos de sucesso, e estão bem consolidados no mercado, e mesmo que a primeira vista possa parecer ligeiramente semelhantes na realidade possuem características que podem fazer a diferença. Estudar esses ambientes proporciona um melhor entendimento da mecânica das plataformas, assim motivando a construção de novos ambientes virtuais que atendam às necessidades do público. O Coursera e o edX foram as plataformas que menos problemas apresentaram durante os testes, mas mesmo estas ainda poderiam melhorar, principalmente no fato de ajuda ao usuário, onde todos os MOOCs deixaram um pouco a desejar. Mais ajuda com ícones e mensagens aparecendo para guiar o estudante e mesmo tutoriais poderiam ser uteis. Pouca estrutura para pessoas com algum tipo de necessidade especial é apresentada, como a áudio descrição, algo que este tipo de sistema poderia apresentar para melhorar a inclusão de os tipos de usuários. 


\section{REFERÊNCIAS}

ALMEIDA, R. Q. 0 leitor navegador. In SILVA, E. T. A leitura nos oceanos da Internet. São Paulo: Cortez, 2003.

ANDRADE, A. F. D.; VICARI, R. M. Construindo um ambiente de aprendizagem à distância inspirado na concepção sociointeracionista de Vygotsky. In: SILVA, Marco (org). Educação On-line. São Paulo: Loyola, 2003. p. 255-270.

BELLONI, M. L. Educação a distância mais aprendizagem aberta. ANPED, GT 16 - Educação e Comunicaç̃̃o. S/d.

BITTENCOURT, D. F. A construção de um modelo de curso "Iato sensu" via Internet - a experiência com o curso de especialização para gestores de instituições de ensino técnico UFSC SENAI. Dissertação de Mestrado. Universidade Federal de Santa Catarina, 1999. Disponível em: <http://www.eps.ufsc.br/ disserta99/denia/>. Acesso: 21 Jun. 2014.

BEVAN, N. Usability is quality of use. Anzai \& Ogawa (eds) Proc. 6thlnternational Conference on Human Computer Interaction, 1995.

BEVAN, N. Usability Issues in web site design. Proceedings of UPA'98, Washigton 1998.

BOYD, D. M.; ELLISON, N. B. Social network sites: Definition, history, and scholarship. Journal of Computer-Mediated Communication, n. 13, p. 210-230, 2008. Disponível em: <http://icmc.indiana. edu/vol13/issuel/boyd.ellison.html>. Acesso em: 21 mai. 2011.

COURSERA. Disponivel em: <https://www.coursera.org/>. Acesso em: 04/11/2014.

EDX. Disponível em: <https://www.edx.org/>. Acesso em: 04/11/2014.

DOTTA, S. Uso de uma Mídia Social como Ambiente Virtual de Aprendizagem.

GOMEZ, Margarida Victoria. Educação em Rede: uma visão emancipadora. São Paulo: Editora Cortez, 2004.

GIGLIO, K.; SOUZA, M.V; SPANHOL, F.J. Redes Sociais e ambientes virtuais de aprendizagem: apontamentos para uma educação em rede. Anais do 19 CIAED-Congresso Internacional ABED de Educação a Distância. Salvador: ABED, set. 2013. Disponível em: <http://www.abed.org.br/congresso2013/cd/141.pdf>. Acesso em: 12 mar. 2014.

HERNÁNDEZ, D. R. Um mundo de médios sin fin. Cambios em aprendizaje, Facebook y apoteosis de las aplicaciones expressivas. In: Facebook y la posuniversidad: sistemas operativos sociales y entornos abiertos de aprendizajes. Fundación Telefónica; Ariel, 2010. p. 183 - 202

LEE, M. J. W; MCLOUGHLIN, C. Harnessing the affordances of Web 2.0 and social software tools: can we finally make "student-centered" learning a reality? Paper presented at the World Conference on Educational Multimedia, Hypermedia and Telecommunications, Vienna, Austria, 2008.

MACIEL, I. M. Educação a Distância: Ambiente virtual: construindo significados. Disponível em: <http:// www.senac.br/BTS/283/boltec283e.htm>. Acesso em: 02 Ago. 2012.

MATTAR, J. 0 uso do second life como ambiente virtual de aprendizagem. Disponivel em: <http://www. comunidadesvirtuais.pro.br/seminario4/trab/jamn.pdf>. Acesso em: 04 mai. 2013.

MAZMAN, G.; USLUES, Y. K. The usage of social networks in educational context. In: Proceedings of 
world academy of science, engineering and technology. Vol. 37, p. 404-407, 2009. McAULEY, A. Massive Open Online Courses: Digital ways of knowing and learning. Elearnspace, 2010. MELO FILHO, I. J. E. A. Percepção social em "EDUCAÇÃO A DISTÂNCIA (EAD)": Identificando necessidades para o LMS Amadeus. Revista Brasileira de Informática na Educação, v. 19, n. 3, p. 29-41, 2011. Miles, M. Huberman, A. Qualitative Data Analysis. 1994.

MIRIADAX. Disponível em: <https://www.miriadax.net/>. Acesso em: 04/11/2014 NIELSEN, J.; MOLICH, R. Heuristic evaluation of user interfaces. Seattle, 1993.

OLIVEIRA, E. A.; TEDESCO, P. i-collaboration: Um modelo de colaboração inteligente personalizada para ambientes de "EDUCAÇ̃̃O A DISTÂNCIA (EAD)". Revista Brasileira de Informática na Educação, v. 18, n. 1, p. 17-31, 2010.

PEREIRA, Alice Theresinha Cybis; SCHMITT, Valdenise; DIAS, Maria Regina Álvares C. Ambientes Virtuais de Aprendizagem. In: PEREIRA, Alice Theresinha Cybis.Ambientes Virtuais de Aprendizagem. São Paulo: Ciência Moderna, 2001. Cap. 1.

PIEROTTI, D. Heuristic Evaluation - A System Checklist. Disponível em: <http://www.stcsig.org/ usability/topics/articles/he-checklist.html>. Acesso em: 15 nov. 2014.

SCHLEMMER, E. A aprendizagem em mundos virtuais: viver e conviver na virtualidade. UNIrevista - Vol. 1, n 2: (abril 2006). Disponível em: <http://www.unirevista.unisinos.br/_pdf/UNlrev_Schlemmer. pdf $>$. Acesso em: 01 mai. 2013.

SIEMENS, G. MOOCs are really a platform. Elearnspace, 25 jul. 2012. Disponivel em $:<$ http://www. elearnspace.org/blog/2012/07/25/moocs-are-really-a-plafform/> Acesso em: 12 mar. 2014.

SOUZA, M. V; et al. Mídias sociais, AVA e MOOCs: reflexões sobre educação em rede.ICBL 2013: International Conference on Interactive Computer aided Blended Learning, Florianópolis, v., n., p. 183190, nov. 2013. Disponível em: <http://www.icbl-conference.org/proceedings/2013/papers/ Contribution62_a.pdf>. Acesso em: 12 mar. 2014.

SOUZA, M. V. Redes informatizadas de comunicação: a teia da rede internacional DPH. São Paulo: Blucher Acadêmico, 2008.

SOUZA, R. K. Análise de Ambientes de Aprendizagem com Características MOOC (massive Open Online (ourse): Uma abordagem qualitativa. Florianópolis, 2014.

STAKE, R. The case study method in social inquiry. In DENZIN, Norman K.

TIFFIN, J, RAJASINGHAM, L. The Global Virtual University, London, New York and Canada: Routledge. 2003.

TÔRRES, J. J. M. Ciência da complexidade: uma nova visão de mundo para a educação. Disponível em: <http://www.otium.net.br/Arquivos/2010-Banner-Julio-Ciencia.pdf>. Acesso em: 12 mai. 2013.

TRIVIÑOS, A. N. S. Introdução à pesquisa em ciências sociais: a pesquisa qualitativa em educação. São Paulo: Atlas, 1987.

UDACITY. Disponível em: <https://www.udacity.com/>. Acesso em: 04/11/2014.

USABILITY.GOV. System Usability Scale. Disponível em: <http://www.usability.gov/how-to-and-tools/ methods/system-usability-scale.html>. Acesso em: 25 out. 2014.

VEDUCA. Disponível em: <http://www.veduca.com.br/>. Acesso em: 04/11/2014. 
Vergara, S. Projetos e Relatórios de Pesquisa em Administração. 8 ed. São Paulo: Atlas, 2007. WALLON, H. As origens do caráter na criança. São Paulo: Nova Alexandria, 1995.

WINCKLER, M.; PIMENTA, M. Avaliação de Usabilidade de Sites Web, Toulouse, 2003. 